\title{
Optics of Electron Beam in the Recycler
}

\author{
A. Burov ${ }^{1}$, G. Kazakevich ${ }^{2}$, T. Kroc ${ }^{1}$, V. Lebedev ${ }^{1}$, S. Nagaitsev ${ }^{1}$, L. \\ Prost $^{1}$, S. Pruss ${ }^{1}$, A. Shemyakin ${ }^{1}$, M. Sutherland ${ }^{1}$, M. Tiunov ${ }^{2}$, A.Warner ${ }^{1}$ \\ ${ }^{1}$ Fermi National Accelerator Laboratory, P .O. Box 500, Batavia IL 60543 \\ ${ }^{2}$ Budker Institute of Nuclear Physics, 630090 Novosibirsk, Russia
}

\begin{abstract}
Electron cooling of $8.9 \mathrm{GeV} / \mathrm{c}$ antiprotons in the Recycler ring (Fermilab) requires high current and good quality of the DC electron beam. Electron trajectories of $\sim 0.2 \mathrm{~A}$ or higher DC electron beam have to be parallel in the cooling section, within $\sim 0.2 \mathrm{mrad}$, making the beam envelope cylindrical. These requirements yielded a specific scheme of the electron transport from a gun to the cooling section, with electrostatic acceleration and deceleration in the Pelletron. Recuperation of the DC beam limits beam losses at as tiny level as $\sim 0.001 \%$, setting strict requirements on the return electron line to the Pelletron and a collector. To smooth the beam envelope in the cooling section, it has to be linear and known at the transport start. Also, strength of the relevant optic elements has to be measured with good accuracy. Beam-based optic measurements are being carried out and analysed to get this information. They include beam simulations in the Pelletron, differential optic (beam response) measurements and simulation, beam profile measurements with optical transition radiation, envelope measurements and analysis with orifice scrapers. Current results for the first half-year of commissioning are presented. Although electron cooling is already routinely used for pbar stacking, its efficiency is expected to be improved.
\end{abstract}

Keywords: Envelope match, angular momentum dominated beam, electron cooling.

PACS: 29.27.Bd, 29.27.Eg, 29.27.Fh

\section{INTRODUCTION}

The Recycler ring (RR) is used for stochastic and electron cooling of $8.9 \mathrm{GeV} / \mathrm{c}$ antiprotons coming from the Accumulator [1]. A layout of the electron cooling line is presented in Fig. 1. The designed beam envelope is shown in Fig. 2, generated by the OptiM code [2, 3]. Main features of the electron cooling line are.

- Electrons are emitted from a thermo-cathode, accelerated and decelerated in an electrostatic accelerator (Pelletron);

- Ideally, electron trajectories in the cooler are straight lines parallel to the axis;

- For focusing purposes, there is $100 \mathrm{G}$ magnetic field in the $20 \mathrm{~m}$ long cooler;

- Magnetic flux at the cathode is equal to the flux at the cooler;

- Matrices Pelletron-Cooler and Cooler-Pelletron are rotation-invariant;

- Possibility for zero dispersion in the return line is foreseen.

\section{ELECTRON ANGLES}

Cooling efficiency strongly depends on the effective angle between the pbars and electrons. To have maximal cooling, the electron rms angle should not exceed the proton angle, at least for the tail protons. 


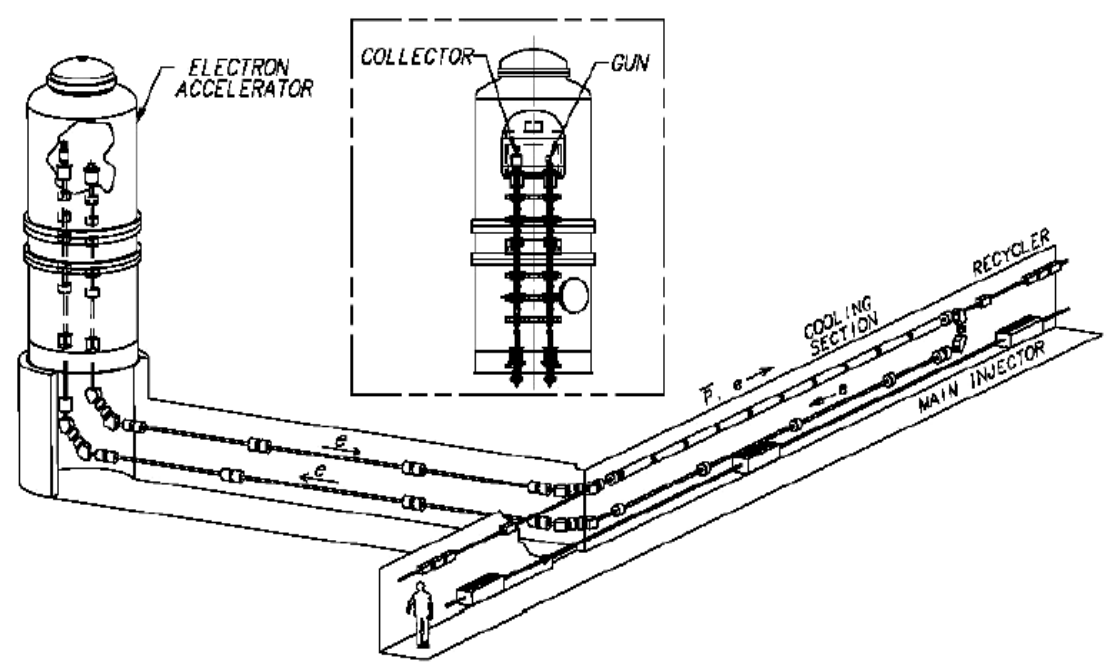

FIGURE 1. Layout of $100 \mathrm{~m}$ long electron cooling line

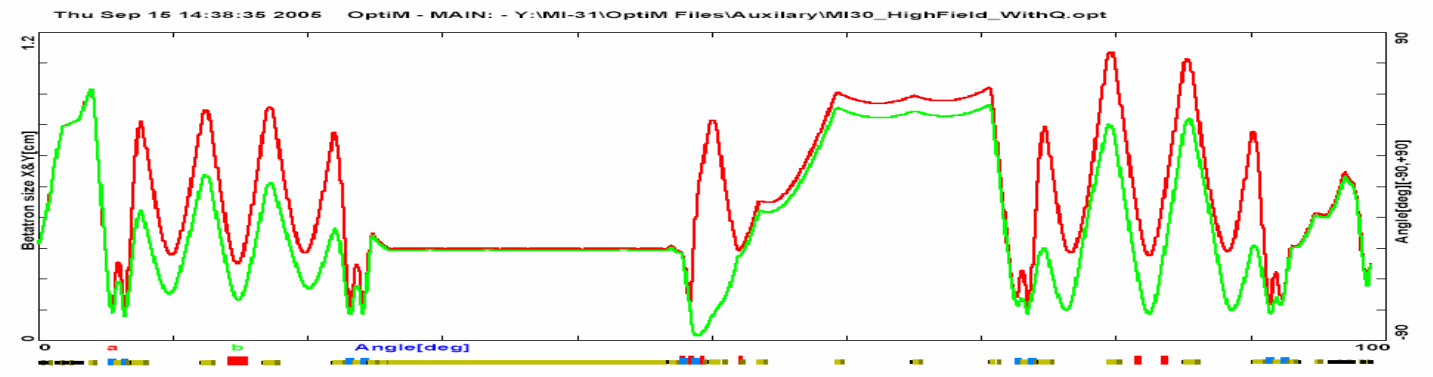

FIGURE 2. Envelope half-axes (design) of the electron cooling line (OptiM simulations). At the bottom, black color is for acceleration and deceleration sections, gold is for solenoids, blue for dipoles, red is for quads.

Assuming 95\% normalized pbar emittance of $5 \mathrm{~mm}$ mrad, the 1D r. m. s. proton angle in the cooler with $30 \mathrm{~m}$ beta-function is $50 \mu \mathrm{rad}$. Electron angles in the cooling section are contributed by the following sources:

- Electron thermal angles; for $0.1 \mathrm{eV}$ of the cathode temperature and equal sizes of the beam at the cathode and the cooler, the $1 \mathrm{D}$ r. m. s. thermal angle of electrons is $50 \mu \mathrm{rad}$.

- Imperfections of the magnetic field in the cooler (static); the last measurements yielded the field r. m. s. angle of $50 \mu \mathrm{rad}$ [4].

- Perturbations from the Main Injector ramps contribute $\sim 40 \mu r a d$.

- Non-linear aberrations of the optical elements in the supply line, mainly doublet solenoids, are estimated to give rise to $\sim 20 \mu \mathrm{rad}$ at the envelope surface; they scale as the offset cubed.

- Non-linearity of the beam angles distribution due to a deviation of the gun optics from the ideal Pierce regime. As a result, the beam has some halo, which optics is far from being similar to the almost linear beam core. Because of that, present halo-matching procedure based on the scraper 
measurements in the cooler leads to the core envelope oscillations with $400 \mu \mathrm{rad}$, according to simulations and the drag force measurements (see more below). This source of the electron angles is currently dominant; hopefully, that envelope mismatch will be significantly reduced in the near future.

\section{MAIN INJECTOR RAMPS CONTRIBUTION}

$\mathrm{RR}$ is located in the same tunnel as the Main Injector (MI). AC magnetic fields excited by the MI ramps give rise to drifts of the electron beam. Although these fields are suppressed by a compensation loop and shielding, some remnant effects still exist. To see how significant they are, the electron orbit was measured during $2 \mathrm{~s}$ of the MI cycle [5] with the sample frequency $700 \mathrm{~Hz}$.

To distinguish the beam signal from the electronic noise, the raw data of 11+11 cooler BPMs were fitted by the helical trajectory for every time sampling point. For sufficient electron current and its modulation depth, the fit was found to be close to the raw signal, so the noise contribution was small. The AC r. m. s. beam angle was calculated then as $40 \mu \mathrm{rad}$, which looks to be small enough for the purposes of cooling.

\section{ENVELOPE MISMATCH}

\section{Envelope Quality}

To find tolerances for the electron envelope mismatch, let us consider an antiproton with an r. m. s. offset $a_{p}$ and angle $\theta_{p}=a_{p} / \beta_{p}$, where $\beta_{p}$ is RR beta-function in the cooler. Cooling of this antiproton would not be reduced by the electron angles if they are smaller than the antiproton angle: $\theta_{e}\left(a_{p}\right)<a_{p} / \beta_{p}$. Assuming the mismatch being linear with the offset, this requirement is identical to $\theta_{e}\left(a_{e}\right)<a_{e} / \beta_{p}$, where $a_{e}$ is the electron beam radius. Taking into account that the electron angle relates to its offset variation $\Delta a_{e}=\theta_{e} \beta_{e}$ with $\beta_{e}=p_{e} c / e B$ as the Larmor beta-function, the matching requirement for maximal cooling can be expressed as

$$
\theta_{e}\left(a_{e}\right)<a_{e} / \beta_{p} \Rightarrow \Delta a_{e} / a_{e}<\beta_{e} / \beta_{p} .
$$

For $B=100 \mathrm{G} \Rightarrow \beta_{e}=160 \mathrm{~cm}$, and $\beta_{p}=30 \mathrm{~m}$, this gives $\Delta a_{e} / a_{e}<0.05$, and with $a_{e}=3.5 \mathrm{~mm}$, it is equivalent to $\Delta a_{e}<0.2 \mathrm{~mm}, \theta_{e}\left(a_{e}\right)<120 \mu \mathrm{rad}$. Note that the linear matching condition (1) does not depend on the pbar emittance.

To match the envelope, two issues have to be known well enough. First, for some optical settings, the envelope parameters somewhere in the line have to be known. Second, properties of the related optical elements have to be known with sufficient accuracy. When both problems are solved, any initial envelope can be matched by a proper change of settings of the well-modeled optical elements. The second problem is being solved by measuring differential trajectories, or responses of the beam trajectory 
on kicks applied by different correctors. The BPM data for the differential trajectories (normally for a set of 4 independent correctors and the energy offset) are fitted by variable optical parameters of the focusing elements. So far, our main approach to the envelope initial condition was based on measurements with 11 orifice scrapers located equidistantly in the cooling section [6]. This sort of measurements is sensitive to the beam halo, not the core. In case of significant non-linearity in the beam angle profiles, the core envelope is not smoothed together with the halo.

\section{Envelope Measurement by Orifice Scrapers}

Every scraper of the cooling section is a copper plate with a round orifice; they are located every $2 \mathrm{~m}$. Normally, all the scrapers are moved out of the chamber. For the envelope measurement, one of the scrapers is moved in, with the center of its orifice approximately coinciding with the chamber axis. Then, the beam is shifted in some direction, and in parallel to the axis, until it starts touching the scraper. The BPM data for the beam center are taken at this point. After that, the beam is shifted in other direction, and everything repeats; normally, 8 directions are used. Then, the entire procedure is repeated for other scrapers.

When the data for all or a sufficient number of the scrapers are taken, the envelope parameters are found in a two-step fitting procedure. At the step number one, the beam ellipse and the scraper offset are found for every scraper involved. At the step number two, initial conditions for the beam envelope at the entrance of the cooler are found by fitting all these ellipses, using 4D phase space coupled optics formalism [2].
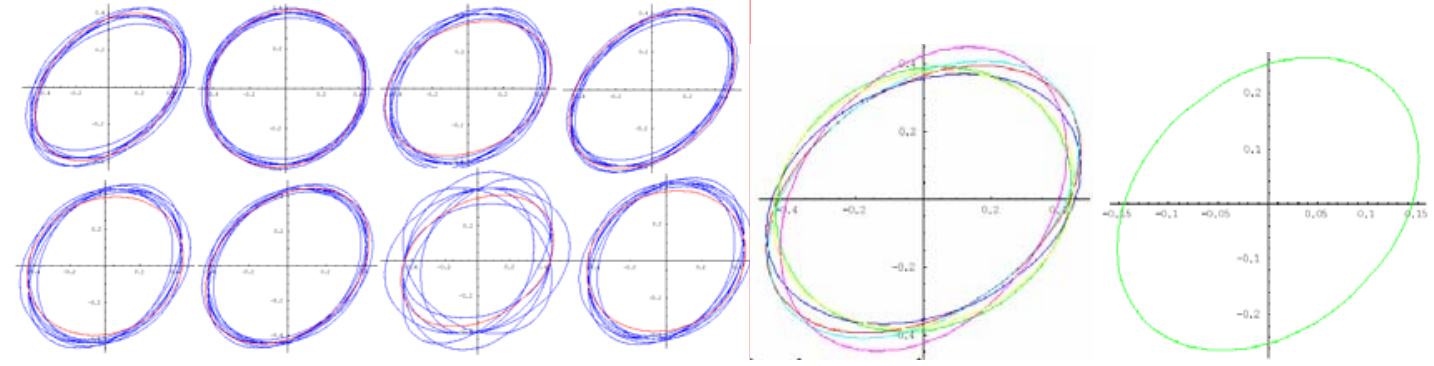

FIGURE 3. Envelope matching the orifice scraper data. On the left, the blue ellipses show the beam cross-section found from the data for every of 8 involved scrapers (in $\mathrm{cm}$ ). Several blue ellipses for every scraper represent the matching error. Red ellipses on the left show the beam envelope in the cooler's magnetic field of $105 \mathrm{G}$, matched to the blue cross-sections. On the center, the found beam envelope is shown for the first 6 scrapers. On the right, the beam angles along the envelope are shown (in mrad), as a parametric plot. Average beam radius is $4.3 \mathrm{~mm}, 2 \mathrm{D} \mathrm{r}$. m. s. angle - $220 \mu \mathrm{rad}$.

Recent results of this analysis are presented in Fig. 3. It is clear that although the envelope is not quite round (which is not necessary), its scalloping along the cooler is rather small; namely, the 2D r. m. s. angle at the envelope has been calculated as 220 $\mu \mathrm{rad}$. Remember that due to the beam non-linearity, this number can significantly underestimate mismatch angles of the beam core. 


\section{OTR Measurements and Simulations}

Other device for the envelope measurements is an optical transition radiation (OTR) image analyzer (see details in Ref [7]). Essentially, this device shows 2D beam density distribution in a transverse to the beam line plane at the OTR location about 2 $\mathrm{m}$ downstream the acceleration exit in the vertical direction. Fig. 4 (left) shows beam profiles detected by OTR and calculated by UltraSAM-BEAM code [8,9] with various settings of a nearest upstream lens. Good agreement between the measurements and calculations convinces us that both of them are essentially correct. Fig. 4 (right) gives the simulation results for beam density, radial and tangential velocity profiles at $\sim 1 \mathrm{MeV}$ of the kinetic energy. Clearly, the velocity profiles are significantly non-linear at the halo. This means that if the beam halo is matched in the cooler, its core would have significant angles. Estimations, based on these results, show that the core envelope angles for matched halo are as high as $\sim 400 \mu \mathrm{rad}$. In other words, for this gun regime, the non-similarity of the beam core and halo is important: when the halo angle is, say, $200 \mu \mathrm{rad}$, the core angle at the nominal radius can be anything between 200 and $600 \mu \mathrm{rad}$. In the near future, either more linear gun regime will be used, or the data analysis will take into account the beam non-linearity.
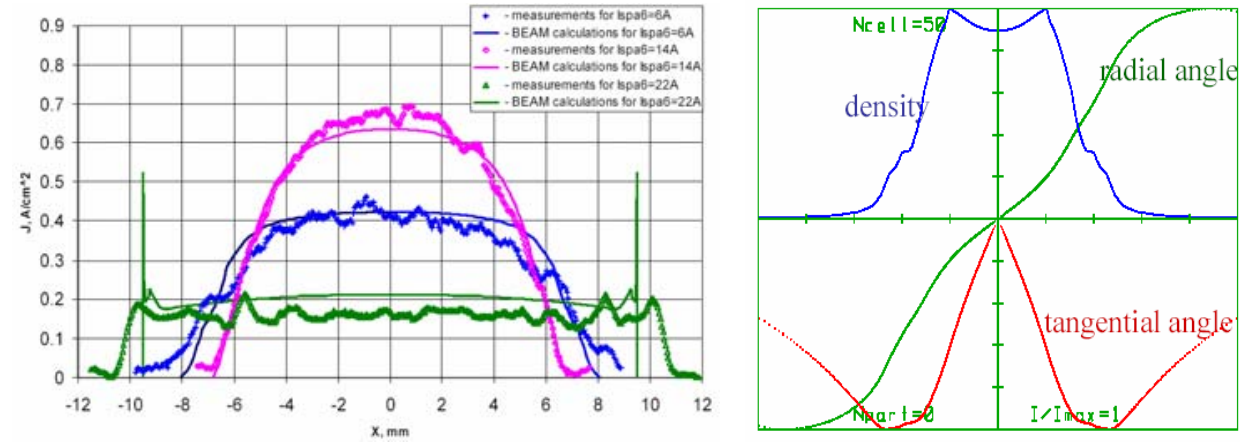

FIGURE 4. On the left: OTR measurements (dots) and UltraSAM-BEAM simulations of the beam transverse density profile for 3 different settings of an upstream lens and $\sim 600 \mathrm{~mA}$ of the beam current. On the right: profiles for the beam density (blue), radial angle (green) and tangential angle (red) at $\sim 1$ $\mathrm{MeV}$ of the beam kinetic energy and $\sim 200 \mathrm{~mA}$ of the current.

\section{REFERENCES}

1. S. Nagaitsev et al., “Antiproton cooling in the Fermilab Recycler”, this conf. (COOL05, Galena, IL, 2005).

2. V. Lebedev and A. Bogazc, "Betatron Motion with Coupling”, e-print JLAB-ACC-99-19 (2001).

3. V. Lebedev, "OptiM”, at http://www-bdnew.fnal.gov/pbar/organizationalchart/lebedev/OptiM/optim.htm .

4. V. Tupikov et al., "Magnetic Field Measurement and Compensation at Fermilab Electron Cooler", this conf.

5. P. Joireman, measurements of Aug. 2005.

6. T. Kroc et al., "Electron Beam Size Measurements in the Fermilab Electron Cooling System”, this conf.

7. A. Warner et al., "OTR Measurements and Modeling of the Electron Beam Parameters at the E-cooling Facility”, this conf.

8. A.V. Ivanov, M.A. Tiunov. "UltraSAM - 2D Code for Simulation of Electron Guns with Ultra High Precision”. Proceeding of EPAC-2002, Paris, 2002, pp.1634-1636.

9. M. A. Tiunov, "BEAM - 2D-Code Package for Simulation of High Perveance Beam Dynamics in Long Systems", Proc. of Space Charge Effects in Formation of Intense Low-Energy Beams, JINR, Dubna, Russia, 1999, pp. 202-208. 
